\title{
Rase Report
}

\section{Primary Intraspinal Extradural Hydatid Cyst of the Dorsal Region: A Case Report}

\author{
Mouhibe Hanane ${ }^{1,}{ }^{*}$, El Mezouari El Mostafa ${ }^{1}, \operatorname{Redouane}_{\text {Rhoukhsi }}{ }^{2}, \operatorname{Redouane~Moutaj}^{1}$ \\ ${ }^{1}$ Department of Parasitology Mycology, Avicenna Military Hospital, Medical School, Cadi Ayyad University, Marrakech, Morocco \\ ${ }^{2}$ Departmentof Radiology, Avicenna Military Hospital, Medical School, Cadi Ayyad University, Marrakech, Morocco
}

Email address:

mouhib.hanane1@gmail.com (M. Hanane)

${ }^{*}$ Corresponding author

\section{To cite this article:}

Mouhibe Hanane, El Mezouari El Mostafa, Redouane Rhoukhsi, Redouane Moutaj. Primary Intraspinal Extradural Hydatid Cyst of the Dorsal Region: A Case Report. Pathology and Laboratory Medicine. Vol. 2, No. 1, 2018, pp. 1-4. doi: 10.11648/j.plm.20180201.11

Received: January 7, 2017; Accepted: April 27, 2017; Published: May 7, 2018

\begin{abstract}
Primarily extradural localization is exceptional posing problems of differential diagnosis even in hydatid endemic countries. We report a case of primary intraspinal extradural hydatid cyst of the dorsal region causing spinal cord compression. The 12-year-old child, living in a rural area with no significant antecedent, presented with gradually increasing three months before his hospitalization a dorsal and lumbarpain installat. He also complained a few days later of numbness and altered sensations in both legs and sphincter disorders. Clinical examination has demonstrated a syndrome of spinal cord compression. Abdominal ultrasound was normal. The diagnosis was an extradural abscess, a dysembryoplastic lesion, or a hydatid localization. A surgical approach was performed in an emergency, consisting of a posterior decompression by laminotomy centered on D12, which resulted in total resection of the cyst repressing and stretching the right root back and the left dural sheath whose hydatid nature was presumptive and confirmed by final histological examination. The immediate consequences were simple.
\end{abstract}

Keywords: Cyst, Echnococcus Granulosus, Marrakech

\section{Introduction}

Hydatidosis, or hydatid cyst, is due to the infestation of man by the tænia of the dog Echinococcusgranulosus. The dog hosts the adult worm in its intestine And the man the larva, in different The organs, but essentially the Liver and lung, in the form of mass Liquid sometimes quite voluminous, Or hydatid cyst.

Spinal hydatid disease is a rare occurrence, characterized by the diversity of its clinical symptomatology and the diagnostic and therapeutic difficulties it raises. Primarily extradural localization is exceptional posing problems of differential diagnosis even in hydatid endemic countries. We report a case of primary intraspinal extradural hydatid cyst of the dorsal region causing spinal cord compression. The presenting symptoms were mostly atypical and the diagnosis was established preoperatively on the basis of magnetic resonance imaging.

Hydatid disease of the spine is caused by the parasite Echinococcusgranulosus, a helminth belonging to the cestode group. Hydatidosis of the bone occurs in $0.5-3 \%$ of all the cases: the vertebral column is involved in $50 \%$ of these $[1,2]$. The disease usually spreads to the spine by direct extension from pulmonary or abdominal infestation and less often begins primarily in the vertebral body [2]. Primary extradural hydatid disease without any systemic foci is extremely rare. It is a common cause of spinal cord compression in endemic areas and the diagnosis remains obscure until symptoms resulting from complications due to root and cord compression appear [3]. Preoperative diagnosis is essential because the rupture and dissemination of cyst may result in anaphylaxis and recurrence.

Through this clinical case our study aims to show the importance of evokes the diagnosis of the extradural hydatic cyst before any cystic lesion in endemic zone. And to discuss possible etiopathogenic hypotheses explaining this localization. Our work also aims to show the importance of MRI which is the exam of choice in the detection of extradural hydatid cyst. 


\section{Observation}

We report a case admitted on March 23, 2016.

The 12-year-old child, living in a rural area with no significant antecedent, presented with gradually increasingthree months before his hospitalization adorsal andlumbarpaininstallat. He also complained a few days later of numbness and altered sensations in both legs and sphincter disorders. Clinical examination has demonstrated a syndrome of spinal cord compression. A dorsal and lumbar MRI performed in the T1 and T2 level, T2 coronal and T2 in level echo in axial sections has objectified an anterior extradural process predominant on the right and driving back the laminated spinal cord back, Bilobate, well limited, hypointense in $\mathrm{T} 1$, hyperintense in $\mathrm{T} 2$, centered on $\mathrm{D} 12$, responsible for a compression of the dural sac with peri-lesional medullary pain (figure 1). CT scan of the dorsolumbar spine in search of bone involvement revealed an intra-dual lesional process centered on D12, bilobed, measuring $25 \times 13 \mathrm{~mm}$ filling the epidural space, driving back the dural sheath without invading it taking finely the contrast medium in Periphery (figure 2). The standard radiological assessment of the spine did not show any bone lesion. Pulmonary radiography was unusual. The biological evaluation was normal outside a discrete hypereosinophilia. Abdominal ultrasound was normal. The diagnosis was an extradural abscess, a dysembryoplastic lesion, or a hydatid localization. A surgical approach was performed in an emergency, consisting of a posterior decompression by laminotomy centered on D12, which resulted in total resection of the cyst repressing and stretching the right root back and the left dural sheath whose hydatid nature was presumptive and confirmed by final histological examination. The immediate consequences were simple.

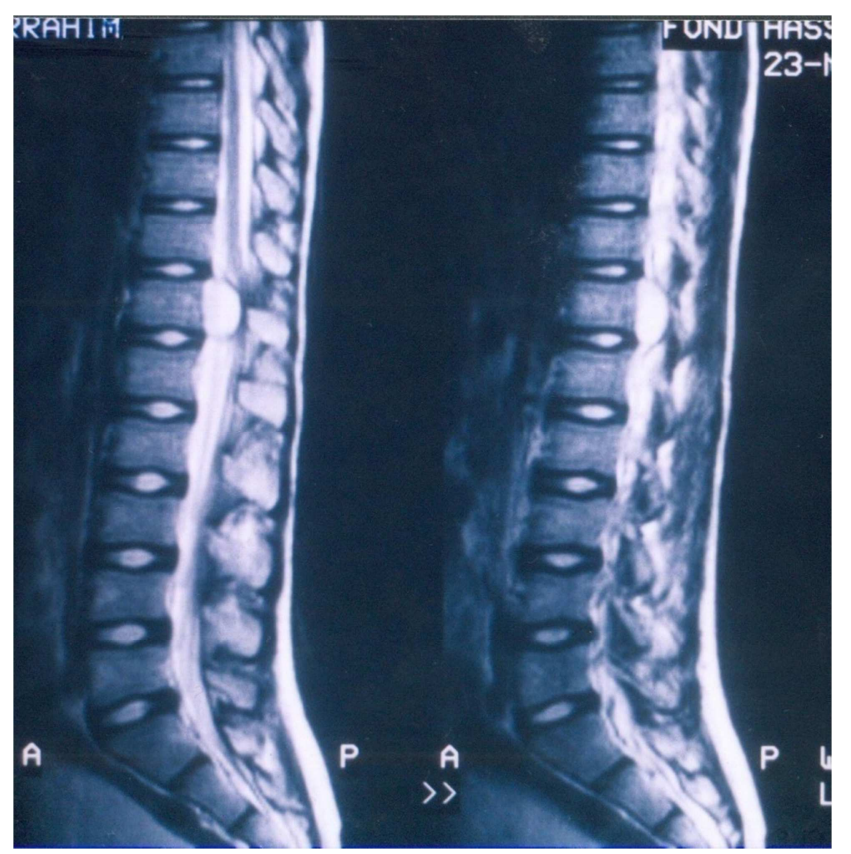

Figure 1. Sagittal sections in T2-weighted sequence, objectifying an anterior extradural process, hyperintense, centered on D12, responsible for compression of the dural sac with peri-lesional medullary pain.

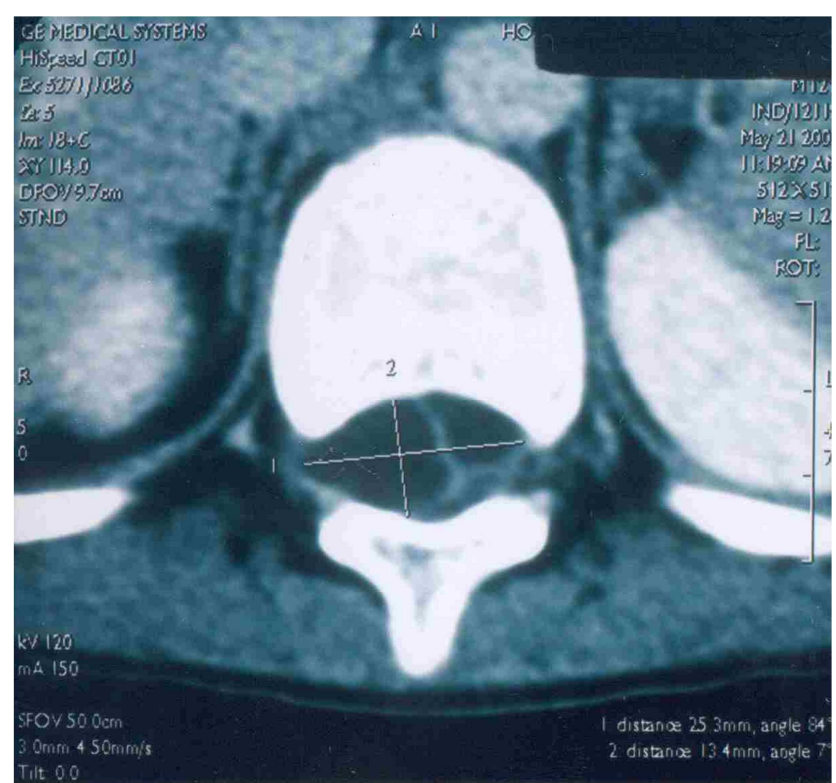

Figure 2. Axial cut in window parenchymateuse after injection of contrast product objectifies a process due to a lesion intracanalairecentered on D12, bilobed, driving back the sheath dural without invading taking it finely the product of contrast in periphery.

A medical treatment based on albendazole was prescribed according to the national standard protocol, at the dose of 400 $\mathrm{mg}$ per day for 28 days repeated three times interspersed with 10 days without treatment, with monthly control of liver function. The evolution was marked by a complete recovery of the deficit after a decline of 11 months.

\section{Discussion}

Hydatid disease has a characteristic geographic distribution and is prevalent in most of the sheep-raising regions of the world $[1,2]$. The infestation occurs either by direct ingestion of parasitic eggs from contact with dogs or indirectly from contaminated water or food [2]. The cysts are transported from the intestinal wall via the blood stream to different organs. As the liver and lungs are the major filters of the body, they are most commonly located in the liver (60-70\%) and lungs (10-15\%); involvement of other organs is rare.

There are many species of Tænia Echinococcus, butonly two Species can reach man:

Echinococcusgranulosus, cosmopolitan and Echinococcusvogeli (in the Americas Rican), while others do not the man. The morphology of scolex and of rings varies according to species.

Echinococcusgranulosus is a small tænia, measuring from 3 to $8 \mathrm{~mm}$ long, formed of a globular head or scolex, 300 microns, equipped with four suction cups, of a projecting rostrum comprising a double crown of hooks. The body is composed of three to four rings, orstrobiles, the last forming a real bag filled with 400 to 800 eggs. These eggs, spherical (about 30 microns) With a thin outer shell and Smooth and a thick and striated inner wall, Contain the hexacanth embryo (three Pairs of hooks). This worm being hermaphrodite, the production of these eggs Is carried out by self-fertilization. 
The hosts vary according to the region: dogs and sheep for E.g. granulosus in Europe, canines and deer in boreal Europe by E.g. borealis and wolves and reindeer for E.g. canadensis, without human injury. Symptoms vary according to the affected organ (liver, lung, heart, kidney, bone, muscles).

Vertebral hydatidosis occurs mostly between $30-50$ years of age.

The extradural localization of the hydatid cyst is a very rare condition and its etiopathogenesis is not clear [19] A few cases have been reported in children also (19.20).

Numerous hypotheses have been proposed to explain this extradural localization. According to Samiy and Zadeh [21] this extradural localization may be due to a spread of the infection from an inapparent bone focus. Cemil et al. [22] raise the hypothesis of venous contamination by the parasite. The sites affected are the thoracic spine in 50\%, lumbar spine in $20 \%$ and sacral spine in $20 \%$ [4]. Cervical involvement is infrequent. The disease usually begins in the vertebral body preferentially in the center of the vertebra. There is a predilection for involvement of the pedicle. The intervertebral disc is usually spared. Perforation of the cortex and periosteum results in extraosseous extension which may be extraspinal or intraspinal [5]. Primary extradural hydatid cyst is rare; primary intramedullary disease extremely rare [5]. Primary extradural hydatid disease of spine can be explained through direct porto-vertebral venous shunts $[6,8]$. The cysts are located epidurally and can be single or multiple. The primary cyst contains daughter cyst and microruptures can give rise to secondary cysts.

It should be noted that intrathecal lesions only become symptomatic at a late stage of the lesion. Once present, the clinical signs are not specific

Clinically, spinal echinococcosis manifests by radicular pain associated with objective sensory and motor disturbances and local tenderness at the level of the involved vertebra. There are no pathognomic signs and symptoms of this disease. For this reason it is often misdiagnosed initially as tuberculosis of the spine, spinal tumor or disc herniation.

The diagnosis is based on a cluster of arguments. Biological examinations are contributory to the diagnosis of the disease. Indeed, at the initial stage of the growth of the cyst and during the accidents of cracking or rupture there is a eosinophilia whose percentage is high [8]. However, this hypereosinophilia is inconstant because it is present only in $25 \%$ of cases [9] and is not specific. The technique of choice is the immunoelectrophoresis by its specificity making it possible to demonstrate the precipitation arc characteristic of hydatidosis [9]. Serology, for its part, has an interest in postoperative monitoring [9].

Radiological exploration makes it possible to define the topography and the relationships of the lesion and to follow the evolution of the lesion [10].

Before any radiological investigation, it is necessary to make simple photographs. The lacunar image of osteolysis is the most characteristic sign of hydatid osteopathy. The gaps are often confluent, variable in size, with blurred boundaries without condensation edges. Sometimes they are multilayered, separated by dividing walls making the image in "cluster of grapes" [10]. Ultrasound can help with positive diagnosis when anatomical conditions allow and shows a fluid collection with central hyperechoic zones. It also makes it possible to look for other hydrostatic visceral sites that are associated with the HVM in $4 \%$ of the cases.

Computed tomography (CT) makes it possible to take stock Complete lesion. Bone damage is reflected in images Uncoated centro-osseous hypodenses after injection of Product of contrast. The scanner makes it possible to appreciate Clear rolling, suffering or rupture of the cortex [10]. The paravertebral collections are also readily Identified, spinal involvement may also be diagnosed and for this purpose an intravenous injection or intrathecal contrast media.

Although CT efficiently demonstrates bony erosion and the extent of the lesion, MR can demonstrate any cord compression throughout the length of the spinal cord and thus is the investigation of choice [5]. On MRI, hydatid cysts appear as well-circumscribed, cystic lesions, with CSF-like signal intensities. The cyst wall is usually thin and regular with no septations. The cysts are hypointense on T1W images [9]. On T2W images they appear hyperintense with sharply defined, hypointense cyst wall which shows mild enhancement following intravenous gadolinium, reflecting the vascularity of the pericyst [10]. There is no contrast enhancement seen after intravenous gadolinium-enhanced MRI either in extradural or intradural hydatid cysts [11]. Extradural spread of hydatid cysts through widened neural foramina into the muscle planes may result in a "bunch of grapes" appearance Jena et al pointed out that the intensity differences in T2W sequences of MRI can also determine the viability of the cyst $[9,12]$.

The treatment is essentially surgical and decompressive laminectomy with total excision of cyst, whenever possible, represents the treatment of choice. However, the invasive nature of the infestation in the spine precludes total removal and therefore permanent eradication [1, 13]. Spinal hydatidosis has been compared to malignant disease of spine $[8,13]$. However, a prolonged and acceptable life still results following surgery. A recurrence risk of $30-40 \%$ has been described [13]. There is a correlation between cyst localization and recurrence. To minimize the risk of recurrence, peroperative use of scolicidal agents like hypertonic saline, $10 \%$ formaldehyde, $0.5 \%$ silver nitrate and povidone iodine has been advocated. Postoperative chemotherapy with mebendazole or albendazole has been recommended for a period of at least three months [3, 7]. Surgery may have to be repeated several times to eradicate the disease completely. Pamir and al 1 reported that $30 \%$ of their patients had previously undergone surgery [1].

If the prophylaxis of wild cycles, which are epidemiological factors of dispersal of hydatidose [17], Is impossibleto realize, that of urban cycles compliance with some measures of elementary general hygiene. The vaccination of dogs with soluble proteins isolated from the protoscolex of $\mathrm{E}$. Granulosus has yielded interesting results [18].

The sustained conjunction of all These measures should 
make it possible to Reduce the human prevalence in A score of years [18]. Hydatidosis is the very type of zoonose which requires joint screening and treatment of individualsand domestic animals as well as the sensitization of populations to insist on hygiene measures prophylactic.

\section{Conclusion}

Bone involvement with Tæniaechinococcus is rare in pathologyts location on the spine is frequent and is the more often primitive and associated with a medullary attack justifying the term vertibromedullaryhydatidosis (HVM). This condition raises difficult diagnostic problems, in addition to the therapeutic and prognostic problems are allotted to it.

The solitary epidural hydatid cyst is a rare entity. However, this diagnosis must be made in front of any cystic lesion in endemic areas. Advances in imaging make MRI the exam of choice in the detection of extradural hydatid cyst.

\section{References}

[1] Pamir MN, Akalan N, Ozgen T, Erbengi A. Spinal hydatid cysts. Surg Neurol 1984; 21: 53-7.

[2] Charles RW, Govender S, Naido KS. Echinococcal infection of the spine with neural involvement. Spine 1988; 13: 47-9.

[3] ROMIG T, DINKEL A, MACKENSTEDT U. The present situation of echinococcosis in Europe. Parasit Int 2006; 55 (Suppl): S187-191.

[4] Morris DL, Richards KS. Hydatid disease. In: Current Medical and Surgical Management. Butterworth. Hieneman Ltd: Oxford; 1992.

[5] Rayport M, Wisoff HS, Zaiman H. Vertebral echinococcosis: Report of case of surgical and biological therapy with review of the literature. J Neurosurg 1964; 21: 647-59.

[6] Abbasioun K, Amirjamshadi A. Diagnosis and management of hydatid cyst of CNS: Hydatid cysts of skull orbit and spine. Neurosurgery 2001; 11: 10-6.

[7] Ley A Jr, Marti A. Intramedullary hydatid cyst. Case report. J Neurosurg 1970; 33: 457-9.

[8] Fiennes AG, Thomas DG. Combined medical and surgical treatment of spinal hydatid disease. J NeurolNeurosurg Psychiatry 1982; 45: 927-30.
[9] Morshed AA. Hydatid disease of spine. Neurochirurgia 1977; 20: $211-5$

[10] Tekkok IH, Benli K. Primary spinal extradural hydatid disease: Report of a case with magnetic resonance characteristics and pathological correlation. Neurosurgery 1993; 33: 320-3.

[11] Gupta S, Rathi V, Bhargava SK. Ventricular primary spinal extradural hydatid cyst - MR appearance. Indian J Radiol Imaging 2002; 12: 271-3.

[12] Hilmani S, El Malki M, Bertal A, Achowi M, Sami A, Onbouknlik A, et al. Lumbar intradural hydatid cyst - Case report. Neurochirurgie 2004; 50: 57-60.

[13] Jena A, Tripathi RP, Jain AK. Primary spinal echinococcosis causing paraplegia- Case report with MR and pathological correlation. AJNR 1991; 12: 560.

[14] Turtas S, Sehrbundt V, Pau A. Long term Results of surgery for hydatid disease of the spine. Surg Neurol 1980; 13: 468-70.

[15] Ozdemir HM, Ogun TC, Tasbas B. A lasting solution is hard to achieve in primary hydatid disease of the spine - Long term results and an overview. Spine 2004; 29: 932

[16] Sapkas GS, Machini TG, Chloros GD, Fountas KN, Themistocleous GS, Vrettakos G. Spinal hydatid disease - A rare but existent pathological entity - A case report and review of literature. South Med J 2006; 99: 178-83.

[17] Sharma NK, Chitkara N, Bakshi N, Guupta P. Primary spinal extradural hydatid cyst. Neurol India 2003; 51: 89-90.

[18] CRAIG PS, MC MANUS DP, LIGHTOWLERS MW, et al. Prevention and Control of cystic echinococcosis. Lancet Infect Dis 2007; 7: 385-94.

[19] Canbolata, Onal, C., Kaya, U., Coban, T. E., 1994. Intracranial extradural hydatid cysts: report of three cases. Surg. Neurol. 41, 230-234.

[20] Braham, E., Bellil, S., Bellil, K., Chelly, I., Mekni, A., Haouet, S., Kchir, N., Khaldi, M., Zitouna, M., 2007. Hydatid cyst of the posterior fossa. Med. Mal. Infect. 37 (5), 281-283

[21] Samiy, E., Zadeh, F. A., 1965. Cranial and intracranial hydatidosis with special reference to roentgen-ray diagnosis. J. Neurosurg. 22, 425-433.

[22] Cemil, B., Tun, K., Gurcay, A. G., Uygur, A., Kaptanoglu, E., 2009. Cranial epidural hydatid cysts: clinical report and review of the literature. Acta Neurochir. 151 (6), 659-662. 